\title{
Desenvolvimento motor de crianças em zonas rurais e urbanas: um estudo comparativo
}

\author{
Motor development of children in rural and urban areas: a comparative study
}

\author{
Renata Rosa Sasso, Susane Graup ${ }^{\mathrm{b}}$, Simone Larac, Rodrigo De Souza Balkd, \\ Eloa Maria dos Santos Chiquettie, Mariana Vieira de Sáa \\ aAcadêmica do Curso de Fisioterapia na Universidade Federal do Pampa (UNIPAMPA), Uruguaiana, RS, Brasil. \\ bEducadora Física. Doutora em Engenharia de Produção. Professora do Curso de Licenciatura em Educação Física na UNIPAMPA, Uruguaiana, RS, Brasil. \\ ' Fisioterapeuta. Doutora em Educação em Ciências. Professora do Curso de Fisioterapia na UNIPAMPA, Uruguaiana, RS, Brasil. \\ ${ }^{d}$ Fisioterapeuta. Doutor em Ciências Biológicas, Professor do Curso de Fisioterapia na UNIPAMPA, Uruguaiana, RS, Brasil. \\ e Fisioterapeuta. Doutora em Ciências do Movimento Humano. Professora do Curso de Fisioterapia na UNIPAMPA, Uruguaiana, RS, Brasil.
}

RESUMO Objetivo: Comparar o desenvolvimento motor de crianças residentes de zonas rurais e urbanas.

Materiais e Métodos: Trata-se de um estudo transversal, quantitativo e descritivo, no qual foram incluídas 153 crianças, de 07 a 10 anos, ambos os sexos, divididas em dois grupos, sendo o Grupo Rural (GR) composto por 76 crianças e o Grupo Urbano (GU) formado por 77 crianças. O desenvolvimento motor foi avaliado através da bateria de testes de avaliação motora - Movement Assessment Battery for Children - segunda edição (MABC-2), que foi desenvolvido para avaliar deficiências motoras de crianças nas faixas etárias de 3 a 16 anos de idade. O MABC-2 contém 3 baterias de testes para respectivas faixas etárias. Para o presente estudo utilizou-se a Banda 2, que avalia crianças de 7 a 10 anos de idade. Esse instrumento é composto por 8 tarefas, 3 tarefas que medem a destreza manual, 2 tarefas medem habilidade com bola e 3 tarefas medem o equilíbrio estático e dinâmico.

Resultados: Houve uma prevalência superior de crianças com atrasos motores no GU (32,5\%) quando comparado ao GR (15,8\%). O GR obteve um melhor padrão motor em relação ao GU nas habilidades motoras de mirar e receber $(p=0,01)$ e de equilíbrio $(p=0,008)$. Quando analisados entre os grupos e conforme o sexo, o grupo feminino urbano foi o grupo com maior frequência de atrasos motores (33,3\%).

Conclusão: As crianças residentes na área rural apresentaram um melhor desenvolvimento motor do que as crianças que vivem na zona urbana.

Palavras-chave: criança; habilidades motoras; equilíbrio postural.

ABSTRACT I Objective: To compare the motor development of children living in rural and urban areas.

Materials and Methods: The cross-sectional, quantitative and descriptive study included 153 children aged 7 to 10 years, both sexes, divided into two groups. The Rural Group (RG) was composed of 76 children and the Urban Group (GU) was composed of 77 children. The motor development was evaluated through a Movement Assessment Battery for Children (MABC), second edition (MABC-2), which was developed to evaluate motor disabilities in children from 3 to 16 years old. The MABC-2 contains 3 test batteries for each age group. We have used the Band 2, which evaluates children from 7 to 10 years old. This method consists of 8 tasks, 3 tasks that measure manual dexterity, 2 tasks measure ball skill, and 3 tasks measure static and dynamic balance.

Results: There was a higher prevalence of children with motor delays in GU (32.5\%) when compared to GR (15.8\%). The GR obtained a better motor pattern in comparison to the $G U$ in the aiming and receiving motor skills $(p=0.01)$ and in the balance $(p=0.008)$. When data was analyzed between the groups and according to gender, the urban female group was the group with the highest frequency of motor delays (33.3\%).

Conclusion: Children living in rural areas had better motor development than children living in urban areas.

Keywords: child; motor skills; postural balance.

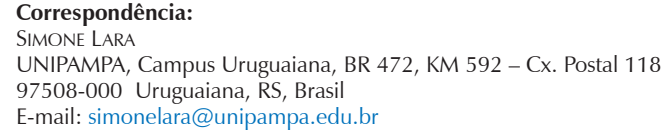




\section{INTRODUÇÃO}

O desenvolvimento motor constitui-se de um processo sequencial, contínuo e multifatorial associado à idade cronológica ${ }^{1}$, que ocorre por meio da associação entre a biologia do indivíduo, as exigências da tarefa de movimento e as circunstâncias do ambiente do aprendizado ${ }^{1}$.

Nesse aspecto de desenvolvimento das capacidades motoras na infância, cabe ressaltar que, aproximadamente aos sete anos de idade, inicia-se uma fase na qual as habilidades estabilizadoras, locomotoras e manipulativas fundamentais são aprimoradas progressivamente, combinadas e elaboradas para aplicação em situações de progressiva demanda ${ }^{1}$. Contudo, para crianças que evidenciam dificuldades motoras, o movimento nesse período pode se configurar um vasto desafio, de modo que determinadas tarefas típicas da infância se tornam muito dificultosas² ${ }^{2}$ e esse comportamento pode estar associado a provável Desordem Coordenativa Desenvolvimental (DCD) ${ }^{3}$.

Considerando que o desenvolvimento na infância pode ser influenciado por diferentes fatores, dentre eles os ambientais ${ }^{4}$, é importante destacar que um meio inapropriado, reprimível ou pouco instigante pode refletir de forma negativa, atrasando o ritmo de desenvolvimento e delimitando as chances de aprendizado da criança ${ }^{5}$. Por outro lado, um ambiente favorável, que permita a experimentação e a exploração da criança é imprescindível para o desenvolvimento dos mais diversos elementos da motricidade, tais como o equilíbrio, a coordenação e o esquema corporal ${ }^{6}$

Nesse contexto, fatores como o ambiente social, a escola, os hábitos e o meio demográfico devem ser levados em consideração por serem potenciais influenciadores sobre o desenvolvimento da criança ${ }^{7}$. Desta forma, $\mathrm{Nave}^{8}$ defende que esse desenvolvimento pode ser mais positivo no meio rural, uma vez que nesse local as capacidades de mobilidade, a exploração corporal, os arremessos e a coordenação em geral são mais favoráveis do que em ambientes urbanos. Contudo, transformações demográficas, socioeconômicas e epidemiológicas, resultados da forçada modernização no campo e do desenvolvimento, vem causando mudanças no perfil do estilo de vida das populações rurais ${ }^{9}$, especialmente na região sul do Brasil ${ }^{10}$

Com base no exposto, o objetivo do presente estudo foi comparar o desenvolvimento motor de crianças de sete a dez anos, que residem em zonas rurais e urbanas, buscando identificar entre esses grupos, se existe diferenças em relação à prevalência de crianças com atrasos motores, quanto aos domínios avaliados (motricidade fina, motricidade ampla e/ ou equilíbrio), e quanto ao sexo.

\section{MATERIAIS E MÉTODOS}

Trata-se de um estudo transversal, quantitativo e descritivo, no qual foram incluídas, por conveniência, crianças de duas escolas públicas de um município no sul do Brasil, sendo uma localizada na zona rural, distante há $40 \mathrm{~km}$ do município mais próximo, e outra situada em zona urbana, situada no centro deste município. A partir da seleção das duas escolas (rural e urbana), os pesquisadores realizaram um sorteio das turmas que seriam incluídas para o estudo, de forma que foram sorteadas 4 turmas em cada escola, incluindo escolares do $2^{\circ}$, $3^{\circ}$ o, 4 을 e $5^{\circ}$ ano.

Para a seleção amostral foram adotados os seguintes critérios de inclusão: a) estar na faixa etária do estudo e; b) estar regularmente matriculado entre o $2^{\mathrm{O}}$ e $5^{\mathrm{O}}$ ano do ensino fundamental, da escola rural ou urbana. Após a seleção amostral, foram excluídas as crianças que apresentassem diagnóstico médico, atestado por meio de laudo médico prévio, de qualquer transtorno de ordem neurológica ou ortopédica, que pudesse interferir na avaliação do desenvolvimento motor.

Todos os critérios éticos foram adotados, e o projeto foi aprovado pelo Comitê de Ética e Pesquisa da instituição local sob o número 1.654.176, em 07/07/2016, e a participação dos escolares na pesquisa foi permitida pelos pais e/ou responsáveis legais por meio da assinatura do Termo de Consentimento Livre e Esclarecido (TCLE).

As crianças participaram de um protocolo de avaliação incluindo os dados de identificação (idade e o ano em que estava cursando na escola), a massa corporal, estatura. Como instrumento de medida para avaliar o desenvolvimento motor dos escolares, foi utilizado o Teste Movement Assessment Battery for Children (MABC) Second Edition (MABC-2), proposto por Henderson et al. ${ }^{11}$, e validado para crianças brasileiras por Valentini et al. ${ }^{12}$. É um instrumento largamente reconhecido na identificação de DCD em crianças com idade entre 3 a 16 anos $^{13}$. A versão brasileira do MABC-2 apresenta altos índices de validade de conteúdo (IVC >0,86), consistência interna $(0,81)$, fidedignidade e estabilidade temporal $(0,82)$, e importante capacidade em discriminar crianças com DCD, em risco e de desenvolvimento típico, permitindo identificar dificuldades específicas na coordenação motora.

O MABC-2 contém 3 baterias de testes para respectivas faixas etárias, nas quais a Banda 1 é destinada para crianças entre 3 e 6 anos de idade; Banda 2 para crianças entre 7 a 10 anos de idade; e a Banda 3 para crianças entre 11 a 16 anos de idade. Cada bateria, por sua vez, possui oito subtestes motores compostos por tarefas de destreza manual, habilidades com bola e equilíbrio estático e dinâmico ${ }^{14}$. No 
Tabela 1. Tarefas para avaliação das habilidades motoras do MABC para a faixa etária de 7 a 10 anos.

\begin{tabular}{|c|c|c|}
\hline Habilidades & Tarefas & Registros \\
\hline Destreza Manual & $\begin{array}{l}\text { Colocar os pinos } \\
\text { Entrelaçar o cordão } \\
\text { Trilha da bicicleta } 2\end{array}$ & $\begin{array}{l}\text { Tempo em segundos } \\
\text { Tempo em segundos } \\
\text { Número de erros }\end{array}$ \\
\hline Habilidades com Bola & $\begin{array}{l}\text { Lançando e recebendo com as duas mãos } \\
\text { Lançando o saco de feijão }\end{array}$ & $\begin{array}{l}\text { Número de recebimentos corretos } \\
\text { Número de acertos no alvo }\end{array}$ \\
\hline Equilíbrio Estático e Dinâmico & $\begin{array}{l}\text { Equilíbrio na prancha } \\
\text { Andando sobre a linha para frente } \\
\text { Saltando em tapetes com um pé }\end{array}$ & $\begin{array}{l}\text { Tempo em segundos } \\
\text { Número de passos corretos } \\
\text { Número de passos corretos }\end{array}$ \\
\hline
\end{tabular}

Fonte: Beltrame et al. ${ }^{15}$ (2017).

presente estudo utilizou-se a Banda 2, devido à idade dos participantes, e essa banda apresenta 3 tarefas que medem a destreza manual, 2 tarefas que medem habilidades com bola e 3 tarefas que medem o equilíbrio estático e dinâmico, conforme Beltrame et al. ${ }^{15}$ (Tabela 1).

Os valores totais adquiridos em cada uma das tarefas motoras são somados e transformados em escores de zero a cinco para cada subteste, sendo que a soma dos escores de cada domínio produz o valor do escore total de prejuízo motor, que é transformado em percentil. Foram aplicados pontos de corte propostos na literatura: escores $\leq 5 \%$ representam um desempenho motor atípico (zona vermelha) identificado como provável Desordem Coordenativa Desenvolvimental (DCD); percentil de $6 \%$ a $15 \%$ considerado desempenho motor suspeito (zona laranja, risco de DCD) e percentil $>16 \%$ considerado como Desenvolvimento Típico (zona verde) ${ }^{14}$.

Cabe ressaltar que a avaliação foi conduzida por duas pesquisadoras previamente treinadas, e a coleta realizou-se nos espaços físicos, cedidos pelas escolas participantes. Os escolares primeiramente recebiam instrução verbal e demonstração das tarefas motoras da bateria. Quando não compreendida a tarefa, nova explicação foi propiciada. A coleta de dados de cada criança levou, em média, 15 minutos.

Para a análise dos dados foi utilizada estatística descritiva, através de medidas de média, desvio padrão e frequências. Para a normalidade dos dados foi utilizado o teste de Kolgomorov-Smirnov que indicou que os dados possuem uma distribuição normal. Para testar as diferenças entre os grupos foi utilizado o teste " $\mathrm{t}$ " não pareado, considerando nível de significância de 0,05.

\section{RESULTADOS}

Fizeram parte do estudo 153 escolares, sendo 77 crianças do grupo urbano - GU (massa corporal: $38,45 \pm 14,87 \mathrm{~kg}$; estatura: $1,37 \pm 0,09 \mathrm{~m}$ e idade: $8,61 \pm 1,1$ anos) e 76 do grupo rural - GR (massa corporal: $34,66 \pm 10,25 \mathrm{~kg}$; estatura: $1,37 \pm 0,08 \mathrm{~m}$ e idade: $8,31 \pm 0,98$ anos).
As características da amostra em relação à distribuição por grupo, sexo, faixa-etária e zona de classificação do desenvolvimento motor estão apresentadas da Tabela 2, sendo possível identificar que, em ambos os grupos houve uma prevalência maior do sexo masculino, e na faixa etária de 08 anos. Quanto às zonas de classificação do desenvolvimento motor, houve uma maior frequência de crianças da área rural na zona verde (em desenvolvimento típico), quando comparado às crianças em áreas urbanas. Ademais, houve um maior percentual de escolares na zona vermelha (com atrasos motores) na área urbana, quando comparada à área rural.

Tabela 2. Distribuição da frequência das variáveis analisadas nos grupos rural e urbano.

\begin{tabular}{|c|c|c|c|c|}
\hline \multirow{2}{*}{ Variável } & \multicolumn{2}{|c|}{ Grupo Urbano } & \multicolumn{2}{|c|}{ Grupo Rural } \\
\hline & $\mathbf{n}$ & $\%$ & $\mathbf{n}$ & $\%$ \\
\hline \multicolumn{5}{|l|}{ Sexo } \\
\hline Masculino & 44 & 57,1 & 41 & 53,9 \\
\hline Feminino & 33 & 42,9 & 35 & 46,1 \\
\hline \multicolumn{5}{|l|}{ Idade } \\
\hline 7 anos & 14 & 18,2 & 18 & 23,7 \\
\hline 8 anos & 25 & 32,5 & 26 & 34,2 \\
\hline 9 anos & 15 & 19,5 & 22 & 28,9 \\
\hline 10 anos & 23 & 29,9 & 10 & 13,2 \\
\hline \multicolumn{5}{|c|}{ Desenvolvimento motor } \\
\hline Zona verde & 42 & 54,5 & 53 & 69,7 \\
\hline Zona laranja & 10 & 13,0 & 11 & 14,5 \\
\hline Zona vermelha & 25 & 32,5 & 12 & 15,8 \\
\hline
\end{tabular}

n: amostra; \%: percentual.

As habilidades motoras avaliadas entre os grupos de crianças estão apresentadas na Tabela 3, sendo possível identificar que houve diferença significativa entre os grupos nas habilidades de mirar e receber $(p=0,01)$ e no equilíbrio $(p=0,008)$, sendo superior nas crianças em meio rural, quando comparados ao meio urbano.

As zonas de classificação do MABC entre os grupos conforme os sexos estão apresentados na Figura 1, 
evidenciando que o grupo com maior frequência de atrasos motores (zona vermelha) foi o urbano feminino $(33,3 \%)$, e a maior prevalência de desenvolvimento motor típico (zona verde) foi o grupo rural masculino $(73,2 \%)$.

A comparação das habilidades motoras intra e intergrupos está presente na Tabela 4. Na comparação intragrupos, os meninos, independente do grupo, apresentaram valores superiores em relação à habilidade motora mirar e receber, quando comparados às meninas $(p=0,01)$. Na comparação intergrupos, foi possível identificar que os meninos da área rural obtiveram valores superiores da habilidade motora mirar e receber quando comparados aos meninos da área urbana $(p=0,02)$. Em relação ao grupo feminino, as meninas da área rural apresentaram valores superiores de equilíbrio, do que as meninas da área urbana $(p=0,02)$.

Tabela 3. Comparação das habilidades motoras do MABC entre os grupos urbano e rural.

\begin{tabular}{lccc}
\hline Variável & $\begin{array}{c}\text { Grupo Urbano } \\
\text { Escore padrão } \\
\text { X } \pm \text { DP }\end{array}$ & $\begin{array}{c}\text { Grupo Rural } \\
\text { Escore padrão } \\
\text { X } \pm \text { DP }\end{array}$ & $\boldsymbol{p}$ \\
Habilidades motoras & & & \\
Destreza Motora & $7,68 \pm 3,13$ & $8,25 \pm 2,78$ & 0,24 \\
Mirar e Receber & $8,31 \pm 2,66$ & $9,59 \pm 3,39$ & $0,01^{*}$ \\
Equilíbrio & $7,31 \pm 2,91$ & $8,82 \pm 3,96$ & $0,008^{*}$ \\
\hline
\end{tabular}

X: dados expressos com valores médios; DP: desvio padrão; *valor significativo.

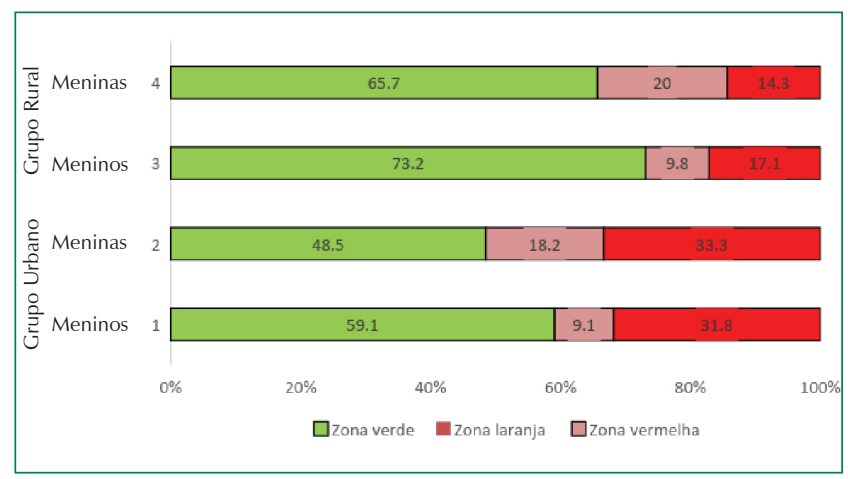

Figura 1. Frequência das zonas de classificação do desenvolvimento motor entre os grupos rural e urbano, conforme o sexo.

\section{DISCUSSÃO}

Ao analisar o nível de habilidades motoras da amostra neste estudo, 54,5\% dos escolares da área urbana e 69,7\% dos escolares da área rural encontram-se na faixa de desenvolvimento motor típico (zona verde). Resultados semelhantes foram encontrados no estudo de Soares et al. ${ }^{16}$, com crianças de ambos os gêneros, na faixa etária de 10 a 12 anos, de instituições públicas e privadas, em um município do interior do Maranhão, em que $60,18 \%$ dos participantes obtiveram uma coordenação motora geral normal. Da mesma forma, Belluzzo et al. ${ }^{17}$ encontraram uma prevalência de $60,9 \%$ de crianças de 8 a 11 anos, de uma rede municipal de ensino da cidade de Ubá, Minas Gerais, em desenvolvimento motor típico.

No presente estudo, identificamos uma maior prevalência de escolares com atrasos motores (zona vermelha) na área urbana (32,5\%), quando comparada à área rural (15,8\%). Cabe destacar que a prevalência de crianças na área vermelha, na área urbana, em nosso estudo, foi superior à prevalência encontrada em outros estudos, como no de Silva e Beltrame ${ }^{18}$ e o de Santos \& Vieira ${ }^{19}$, com crianças da área urbana e da mesma faixa-etária. Os primeiros autores encontraram uma prevalência de $11,1 \%$ de escolares na zona vermelha, em uma escola pública na cidade de São José, Santa Catarina. O segundo trabalho encontrou uma prevalência de $11,4 \%$ de escolares na zona vermelha, avaliadas em instituições educacionais públicas no município de Maringá, Paraná. É importante colocar que, nos estudos citados $^{18,19}$, as crianças realizavam educação física escolar, diferentemente das crianças do atual estudo (tanto do grupo rural quanto urbano), em que não eram ofertadas aulas de educação física nos anos iniciais da escola, o que pode explicar tais diferenças encontradas.

Os resultados de nosso trabalho evidenciaram que, quando as habilidades motoras foram comparadas entre os grupos, o equilíbrio e a motricidade ampla relacionada às habilidades com bola, foram superiores nas crianças em meio rural, quando comparadas ao meio urbano. Por outro lado, o estudo de Souza et al. ${ }^{20}$ não encontrou diferenças quanto às

Tabela 4. Valores descritivos dos escores das categorias e total do teste de Desenvolvimento motor nos grupos rural e urbano.

\begin{tabular}{|c|c|c|c|c|c|c|c|c|}
\hline \multirow[b]{2}{*}{ Variável } & \multicolumn{2}{|c|}{ Grupo Urbano } & \multirow[b]{2}{*}{$p 1$} & \multicolumn{2}{|c|}{ Grupo Rural } & \multirow[b]{2}{*}{$p 1$} & \multicolumn{2}{|c|}{ p2 } \\
\hline & $\begin{array}{c}\text { Meninos } \\
\mathrm{X} \pm \mathrm{DP}\end{array}$ & $\begin{array}{c}\text { Meninas } \\
X \pm D P\end{array}$ & & $\begin{array}{c}\text { Meninos } \\
\mathrm{X} \pm \mathrm{DP}\end{array}$ & $\begin{array}{c}\text { Meninas } \\
X \pm D P\end{array}$ & & Meninos & Meninas \\
\hline Destreza Motora & $7,1 \pm 2,6$ & $8,4 \pm 3,6$ & 0,07 & $7,9 \pm 3,1$ & $8,6 \pm 2,4$ & 0,31 & 0,19 & 0,81 \\
\hline Mirar e Receber & $8,9 \pm 2,6$ & $7,4 \pm 2,6$ & $0,01^{*}$ & $10,5 \pm 3,6$ & $8,5 \pm 2,8$ & $0,01^{*}$ & $0,02 *$ & 0,10 \\
\hline Equilíbrio & $7,6 \pm 2,7$ & $6,9 \pm 3,0$ & 0,31 & $8,8 \pm 4,2$ & $8,8 \pm 3,7$ & 0,77 & 0,12 & $0,02 *$ \\
\hline
\end{tabular}

$\mathrm{X}$ : dados expressos com valores médios; DP: desvio padrão; $p 1$ : diferença entre meninos e meninas intragrupo; p2: diferença entre os sexos intergrupos; * valor significativo. 
habilidades manuais, com bola e de equilíbrio, entre crianças portuguesas residentes de zonas rural e urbana. Porém, indo ao encontro dos dados de nosso estudo, Capellini et al. ${ }^{21}$ identificaram em uma pesquisa com crianças italianas de 6 a 7 anos de idade, que as residentes em meio urbano apresentavam um equilíbrio inferior, quando comparadas ao das crianças em áreas rurais.

Com base em nossos resultados, foi possível destacar a influência do fator ambiental no contexto do desenvolvimento infantil, e nesse sentido, alguns autores como Pedrosa et al. ${ }^{22}$ e Brown et al. ${ }^{23}$ defendem o ambiente rural como o meio demográfico mais favorável ao desenvolvimento motor das crianças. Pedrosa et al. ${ }^{22}$ reiteram que o estilo de vida é mais ativo nas zonas rurais, principalmente pelo favorecimento do ambiente, no qual as crianças desenvolvem com mais facilidade atividades cotidianas que estão associadas ao movimento, como andar de bicicleta, correr, dentre outras. Ademais, Brown et al. ${ }^{23}$ evidenciaram que crianças residentes no meio rural estão engajadas em uma ampla gama de atividades e apresentam uma maior frequência nessas atividades, do que as crianças que vivem em meios urbanos.

Sob esse olhar, as crianças do meio rural, diferentemente das residentes em meio urbano, utilizam frequentemente, como meio de transporte, o cavalo. Nesse cenário, é possível que essa característica possa interferir positivamente sobre as habilidades motoras das crianças avaliadas, uma vez que o andar a cavalo, propicia diversos movimentos multidirecionais e tridimensionais, transmitindo ao corpo humano uma sequência de informações que favorecem a coordenação viso-motora, coordenação motora global, lateralidade e o equilíbrio ${ }^{24}$.

Já nos grandes centros urbanos, devido ao aumento da violência, do fluxo de carros e da escassez de ambientes próprios e acessíveis ao lazer infantil, Oliveira ${ }^{25}$ relata que a autonomia e a mobilidade das crianças têm sofrido interferência. $\mathrm{O}$ autor reitera ainda que esta situação priva a criança de adquirir a imunidade que lhe é atribuída pelo brincar espontâneo e pelo vínculo com o meio em que vive, contribuindo para uma menor capacidade de defesa, dificuldades de adaptação e autonomia para interagir com as novas experiências ofertadas pelo ambiente. Contextualizando com os dados do presente estudo, é importante destacar a ausência de espaços físicos voltados à prática de atividade física/esportiva e ao lazer, nas proximidades da escola urbana, fator que também contribui, conforme já exposto, para um desenvolvimento motor infantil desfavorável.

Para Chaves ${ }^{26}$ no aspecto atual da sociedade, a evolução da tecnologia e dos meios de comunicação têm gerado grandes mudanças nas relações humanas. Tais modificações tecnológicas, mais expressivas no ambiente urbano em comparação ao ambiente rural, interferem também no modo da criança brincar, uma vez que a relação das crianças com jogos, brincadeiras e brinquedos tradicionais vem perdendo preferência para dispositivos tecnológicos, como celulares, videogames, computadores, tablets, televisores e brinquedos eletrônicos. Corroborando, Paiva \& $\operatorname{Costa}^{27}$, alertam que o desenvolvimento e amadurecimento afetivo, físico, cognitivo e social das crianças podem ser prejudicados pela substituição de brincadeiras como pega-pega, escondeesconde, jogar bola - atividades que envolvem movimento físico - por jogos eletrônicos e utilização da tecnologia de forma indiscriminada pelas crianças.

Sendo assim, o contexto em que as crianças da área urbana vivem atualmente, incluindo um estilo de vida mais inativo, com menor exploração e interação ambiental, aliado as suas novas formas de brincar, podem acarretar um ambiente desfavorável para o desenvolvimento motor infantil. Por outro lado, as crianças do meio rural exploram com maior propriedade o ambiente em que vivem, adquirindo maiores vivências e diferentes experiências motoras, através de brincadeiras mais ativas fisicamente, e tais fatores contribuem para o desenvolvimento motor de forma natural. Esses aspectos descritos podem explicar, em parte, a maior prevalência de escolares com atrasos motores (zona vermelha), na zona urbana no presente estudo, comparada com os escolares em meio rural.

Nas análises quanto aos grupos e conforme o gênero, percebemos que o grupo com maior frequência na zona vermelha foi o urbano feminino (33,3\%), seguido do urbano masculino (31,8\%). Ademais, em uma análise intragrupo, as habilidades de mirar e receber foram superiores nos meninos, quando comparadas às meninas, em ambos os grupos. Já na análise intergrupos, os meninos da área rural obtiveram valores superiores na habilidade motora de mirar e receber quando comparados aos meninos da área urbana, enquanto que as meninas da área rural apresentaram valores superiores nas habilidades de equilíbrio, do que as meninas da área urbana.

Em conformidade com esses achados, o estudo com crianças brasileiras feito por Spessato et al. ${ }^{28}$ demonstrou que as meninas foram menos competentes que os meninos, nas idades de 7 a 10 anos, nas habilidades de locomoção e de controle de objeto. Belluzzo et al. ${ }^{17}$, ao analisarem 69 crianças brasileiras, de ambos os sexos e com idade entre 8 e 11 anos, verificaram que em tarefas de equilíbrio (salto mono pedal e lateral) e de coordenação motora geral, houve diferenças significativas em relação a variável sexo, onde os meninos compreenderam valores superiores em relação as meninas. 
Nesse sentido, acredita-se que existam fatores culturais e comportamentais que possam interferir nas questões relacionadas com as diferenças de habilidades motoras conforme o sexo. Por questões culturais, os meninos são mais estimulados às práticas motoras como jogar futebol e andar de bicicleta do que as meninas, e as consequências desse perfil cultural repercutem nos achados de estudos que atribuem habilidades motoras fundamentais superiores para o sexo masculino ${ }^{28}$

Sob esse olhar, Goellner ${ }^{29}$ reitera, em uma perspectiva histórica, que não são iguais as condições de acesso e participação das mulheres, se comparado aos homens, no campo das práticas corporais e esportivas, seja no esporte de rendimento, no lazer, na educação física escolar, na visibilidade conferida pela mídia, entre outras. Tucker et al. ${ }^{30}$ alertam para uma maior atenção às meninas nesse sentido, pois diversas vezes por questões culturais, estas deixam de ter acesso às mesmas experiências motoras propiciadas aos meninos, podendo conduzir a perdas quanto à aprendizagem de habilidades motoras básicas, durante a fase considerada fundamental para o seu desenvolvimento, entre 5 a 10 anos.

Nessa perspectiva, as meninas da área urbana obtiveram maiores percentuais de atrasos motores (zona vermelha) que os demais grupos avaliados, indicando a relevância de obter um olhar mais atento para esta classe específica, com propostas e medidas objetivas, que possam modificar tal realidade. Portanto, com base nesse perfil de risco, devido às razões culturais e de estilo de vida, se faz necessárias ações preventivas, no âmbito familiar e escolar, para melhorar as habilidades motoras dessas crianças. Este fator perpassa por uma série de reflexões, incluindo os hábitos de vida infantis, especialmente relacionados ao acesso a dispositivos eletrônicos por longos períodos durante o dia e ao incentivo a preferência por brincadeiras mais ativas, a importância de incluir aulas de educação física nos anos iniciais do ensino público, a inserção de projetos multidisciplinares junto aos profissionais de educação física e fisioterapia para promover a saúde do escolar, acompanhando o crescimento e o desenvolvimento corporal das crianças e adolescentes, dentre outras.

Considerando os múltiplos aspectos que influenciam o desenvolvimento motor da criança, a não investigação de questões importantes como o nível socioeconômico da família e hábitos de vida das crianças (alimentação, nível de atividade física habitual), constituem limitações desse estudo, uma vez que poderiam permitir comparações mais conclusivas entre os grupos.

Por fim, as crianças residentes na área rural apresentaram um melhor desenvolvimento motor do que as crianças que vivem na zona urbana, em relação às habilidades com bola e de equilíbrio. Ademais, houve um maior percentual de escolares com atrasos motores (zona vermelha) na área urbana, quando comparada à área rural, indicando que o contexto ambiental interfere nas habilidades motoras na infância. Quando observado os achados conforme o sexo, as meninas do meio urbano apresentaram maior prevalência de atrasos motores em relação aos demais grupos, e, portanto, sugere-se um olhar para medidas de promoção de atividades motoras voltadas especificamente para essa população.

\section{REFERÊNCIAS}

1. Gallahue DL, Ozmun JC, Goodway JD. Compreendendo o desenvolvimento motor: bebês, crianças, adolescentes e adultos. 7aㅡ ed. Porto Alegre: AMGH; 2013.

2. Silva JAO, Dantas LE, Cattuzzo MT, Walter C, Moreira CRP, Souza CJF. Teste MABC: aplicabilidade da lista de checagem na Região Sudeste do Brasil. Rev Port Ciênc Desporto. 2006;6(3):356-61. https://doi.org/10.5628/rpcd.06.03.356

3. Diagnostic and statistical manual of mental disorders. 4th ed. Washington: DC: American Psychiatric Association; 1994.

4. Nazario PF, Peres LW, Krebs RJ. A influência do contexto no comportamento motor: Uma revisão. Rev Digital EFDeportes.com. 2011;152(15):1.

5. Spessato BC, Valentini NC, Krebs RJ, Berleze A. Educação infantil e intervenção motora: um olhar a partir da teoria bioecológica de Bronfenbrenner. Movimento. 2009;15(4):147-73.

6. Papst J, Marques I. Avaliação do desenvolvimento motor de crianças com dificuldade de aprendizagem. Rev Bras Cineantropom Desempenho Hum. 2010;12(1):36-42.

7. Venetsanou F, Kambas A. Environmental factors affecting preschoolers' motor development. Early Child Educ J. 2010;37: 319-27. https://doi.org/10.1007/s10643-009-0350-z

8. Nave MLJ. A criança, o meio e o perfil psicomotor [dissertação]. Castelo Branco: Instituto Politécnico de Castelo Branco; 2010.

9. Hoehr CF, Reuter CP, Tornquist L, Nunes HMB, Burgos MS. Prevalência de obesidade e hipertensão arterial em escolares: estudo comparativo entre escolas rurais do município de Santa Cruz do Sul/RS. Rev Epidemiol Control Infect. 2014;4(2):122-6. https:// doi.org/10.17058/reci.v4i2.4772

10. Witeck GA, Franz LBB, Busnello MB, Battisti IDE, Marchi D, Berlezi EM, Cargnin D, Dal Ri RK. Índices antropométricos e fatores de risco cardiovascular entre mulheres residentes em uma área rural do estado do Rio Grande do Sul. Sci Med. 2010;20(4):282-8.

11. Henderson S, Sugden DA, Barnett A. Movement assessment battery for children. 2nd ed. San Antonio: Harcourt Assessment; 2007.

12. Valentini NC, Ramalho MH, Olivei $\neg$ ra MA. Movement assessment battery for children-2: translation, reliability, and validity for Brazilian children. Res Dev Disabil. 2014;35(3):733-40. https://doi. org/10.1016/j.ridd.2013.10.028

13. Toniolo CS, Capellini SA. Transtorno do desenvolvimento da coordenação: revisão de literatura sobre os instrumentos de avaliação. Rev Psicopedag. 2010;27(82):109-16. 
14. Valentini NC, Coutinho MTC, Pansera SM, Santos VAP, Vieira JLL, Ramalho $\mathrm{MH}$, Oliveira MA. Prevalência de déficits motores e desordem coordenativa desenvolvimental em crianças da região Sul do Brasil. Rev Paul Pediatr. 2012;30(3):377-84. https://doi. org/10.1590/S0103-05822012000300011

15. Beltrame TS, Capistrano R, Alexandre JM, Lisboa T, Andrade RD, Felden EPG. Prevalência do Transtorno do Desenvolvimento da Coordenação em uma amostra de crianças brasileiras. Cad Bras Ter Ocup. 2017;25(1):105-13. https://doi.org/10.4322/0104-4931. ctoAO0777

16. Soares NIS, Leone ID, Costa VB, Silva VF, Cabral PUL, Vieira CMS, Madeira FB. Coordenação motora em escolares: relação com a idade, gênero, estado nutricional e instituição de ensino. Biomotriz (Online). 2014;8(1):36-48.

17. Belluzzo PR, Rufino MB, Cabral JFR, Costa JCM, Oliveira RAR, Soares LA, Ferreira EF. Desempenho motor de escolares matriculados nos anos iniciais do ensino fundamental. RBPFEX. 2016;10(62):773-81.

18. Silva J, Beltrame TS. Indicativo de transtorno do desenvolvimento da coordenação de escolares com idade entre 7 e 10 anos. Rev Bras Ciênc Esporte. 2013;35(1):3-14. https://doi.org/10.1590/S010132892013000100002

19. Santos VAPD, Vieira JLL. Prevalência de desordem coordenativa desenvolvimental em crianças com 7 a 10 anos de idade. Rev Bras Cineantropom Desempenho Hum. 2013;15(2):233-42. https://doi. org/10.5007/1980-0037.2013v15n2p233

20. Souza C, Ferreira L, Catuzzo MT, Corrêa UC. O teste ABC do movimento em crianças de ambientes diferentes. Rev Port Cien Desp. 2007; 7(1):36-47.

21. Cappellini AC, Mancini S, Zuffellato S, Bini F, Polcaro P, Conti AA, Molino LR, Macchi C. Environmental effects on school age child psychomotricity. Minerva Pediatr. 2008;60(3):277-84.
22. Pedrosa OP, Pereira ACB, Silva AC, Pinho ST. Aptidão física de escolares da zona urbana e da zona rural da cidade de Porto Velho/ RO. An Sem Educa. 2010;1(1):181-95.

23. Brown T, O'Keefe S, Stagnitti K. Activity preferences and participation of school-age children living in urban and rural environments. Occup Ther Health Care. 2011;25(4):225-39. https://doi.org/10.3 109/07380577.2011.589889

24. Barbosa GO, Munster, MA. O efeito de um programa de equoterapia no desenvolvimento psicomotor de crianças com indicativos de transtorno de déficit de atenção e hiperatividade. Rev Bras Educ Espec. 2014;20(1):69-84. https://doi.org/10.1590/ S1413-65382014000100006

25. Oliveira C. O ambiente urbano e a formação da criança. São Paulo: Aleph; 2004.

26. Chaves ICG. Tecnologia e infância: um olhar sobre as brincadeiras das crianças. [trabalho de conclusão de curso]. Maringá: Universidade Estadual de Maringá; 2014.

27. Paiva NMN, Costa JS. A influência da tecnologia na infância: desenvolvimento ou ameaça? Portal dos psicólogos [periódico online]. 2015 [capturado 2018]. Disponível em: http://www. psicologia.pt/artigos/textos/A0839.pdf

28. Spessato BC, Gabbard C, Valentini N, Rudisill M. Gender differences in Brazilian children's fundamental movement skill performance. Early Child Dev Care. 2013;183(7):916-23. https://doi.org/10.108 0/03004430.2012.689761

29. Goellner SV. Mulher e esporte no Brasil: entre incentivos e interdições elas fazem história. Pensar Prát. 2005;8(1):85-100.

30. Tucker P. The physical activity levels of preschool-aged children: a systematic review. Early Child Res Q. 2008;23:547-58. https://doi. org/10.1016/j.ecresq.2008.08.005 